\title{
Conséquences juridiques et financières de l'accident du salarié sous l'emprise d'une substance psychoactive
}

\section{Juridical and financial consequences of the accident of an employee under the influence of a psychoactive substance}

Laurent DAVENAS ${ }^{(1)}$, Gilbert PÉPIN*(2)

(1) Haut Avocat Général à la Cour de Cassation, 5, rue Gustave Courbet - 75016 PARIS (2) Laboratoire TOXLAB, 7, rue Jacques Cartier - 75018 PARIS

* Auteur à qui adresser la correspondance : Gilbert PÉPIN, Laboratoire TOXLAB, 7, rue Jacques Cartier 75018 PARIS - Tél : 0158592800 - Fax : 0158592801 - e-mail : labtoxlab@aol.com

(Reçu le $1^{\text {er }}$ février 2002 ; accepté le 20 février 2002)

\section{RÉSUMÉ}

Dans trois cas, nous étudierons la responsabilité de salariés sous l'emprise de produits psychoactifs (alcool et cannabis par exemple) pendant leur activité professionnelle. Les principes retenus à ce jour par les juridictions sont que la faute exclusive de l'auteur d'un grave accident est une source d'exonération de responsabilité, particulièrement pénale, pour un employeur et souvent, une cause de réduction, voir de suppression de l'indemnisation à laquelle peuvent prétendre la victime ou ses ayants-droits. Toutefois, la preuve du caractère unique et exclusif de la faute est très rarement retenue par la Jurisprudence. En effet, la tendance contemporaine accentue la volonté sociale d'une indemnisation la plus large possible des victimes.

\section{MOTS-CLÉS}

Accidents du travail, substances psychoactives, faute, responsabilité pénale.

\section{SUMMARY}

In three different cases we study the responsibility of employees under the influence of psychoactive substances (alcohol, cannabis for example) during their professional activity. The principles retained to date by the jurisdictions are that the exclusive fault of the author of a serious accident is a source of exoneration of responsibility, particularly regarding the penal liability, of an employer and often, a cause of reduction, to see suppression of indemnity to which can claim the victim or its have-rights. However, the proof of the single and exclusive character of the fault is very seldom retained by the Jurisprudence. As a matter of fact, the current tendency accentuates the social willing of the broadest as possible compensation for the victims.

\section{KEY-WORDS}

Workplace injuries, psychoactive substances, fault, penal responsibility. 


\section{A propos de trois cas}

1 - Le conducteur d'une automobile, salarié, percute à grande vitesse un pilier en béton : trois morts et un blessé grave. Son alcoolémie est élevée. Sa famille touchera-t-elle une indemnité ? Les familles de ses passagers pourront-elle réclamer à son employeur une indemnité, la cause du décès de celle-ci étant imputée à un chauffeur ivre?

2 - Le conducteur d'un gros engin de chantier écrase un de ses collègues contre un mur. L'analyse sanguine montre qu'il était sous l'influence de cannabis. Qui est responsable : le conducteur salarié ou le chef de l'entreprise ?

3 - Sur un chantier d'un immeuble en construction, un ouvrier tombe du $7^{\text {ème }}$ étage. Il était sous l'influence de l'alcool et du cannabis. Sa conjointe touchera-t-elle une indemnité de l'entreprise qui l'employait ?

\section{Aspects juridiques et finan- ciers}

Nous développerons l'incidence de l'ivresse ou de la consommation de cannabis d'un employé sur les responsabilités et les indemnités consécutives à un grave accident.

Les accidents survenant par le fait ou à l'occasion du travail à toute personne salariée entraînent l'application de la législation professionnelle spécifique permettant une meilleur indemnisation des victimes et de leurs ayants-droits au titre des accidents du travail par la Sécurité Sociale et, éventuellement, par l'employeur pour le préjudice complémentaire subi.

La question qui se pose est la suivante : dans quelle mesure la faute commise par l'employé qui cause ou subit un accident après consommation d'alcool ou de cannabis sera-t-elle retenue ?

Le régime juridique se caractérise par :

- une responsabilité civile de plein droit de l'employeur entraînant une indemnisation automatique des victimes et des ayants-droits par la Sécurité Sociale - Loi du 30 octobre 1946 - du préjudice professionnel, outre l'indemnisation du préjudice complémentaire en cas de faute inexcusable de l'employeur.

- une réduction de l'indemnisation de la victime et de ses ayants-droits si elle a commis, elle-même, une faute inexcusable - article L 453-1 du Code de la Sécurité Sociale.

- l'absence de toute prestation ou indemnité au titre de la législation sur les arrêts de travail si l'accident résulte de la faute intentionnelle de la victime (L 453-1 du
Code de la Sécurité Sociale).

- une responsabilité pénale de l'employeur du fait d'atteinte involontaire à la personne d'autrui (221-6, 22219, 222-20, R 622-1, R 625-3, R 625-2 du Code Pénal) ou du fait de mise en danger d'autrui (121-3 du Code Pénal) (223-1 du Code Pénal) qui ne peut que difficilement s'exonérer en démontrant :

(outre la force majeure (contrainte : 122-2 du Code Pénal) ou la délégation de pouvoir) :

- le fait d'un tiers ou de la victime : mais seulement s'il s'agit de la cause exclusive et unique du dommage.

Les fautes sont de trois types : inexcusable, intentionnelle et exclusive. Chacune entraîne des conséquences particulières sur l'indemnisation des victimes et méritent d'être examinées, au regard de la faute commise par le salarié se trouvant en état d'ivresse ou sous l'emprise de cannabis et causant ou subissant lui-même un grave accident. De manière générale, l'ivresse ou la prise de drogue sur les lieux ou pendant le travail constitue une faute grave justifiant un licenciement immédiat. Mais pour autant, cette faute est-elle une faute intentionnelle ou inexcusable et quelle est son influence sur l'indemnisation?

Le principe retenu par nos juridictions est le suivant :

- l'employeur qui dispose d'une obligation de surveillance et du pouvoir disciplinaire ne doit pas tolérer une telle situation : en conséquence, l'accident survenant dans les temps et lieu du travail à un salarié ivre (ou sous l'emprise du cannabis) relève de la législation professionnelle permettant l'indemnisation spécifique à titre de législation des Accidents de Travail.

- seule la preuve que l'employé s'est enivré ou drogué dans des circonstances telles qu'il est soustrait à l'autorité de l'employeur supprimera cette indemnisation, laquelle sera simplement réduite à défaut d'une telle preuve.

La Cour d'Appel de Versailles ( $5^{\text {ème }}$ Chambre Sociale 28/11/95. Société OTIS C/CPAM) a ainsi jugé que : «la présomption d'imputabilité selon laquelle tout accident survenu au temps et au lieu du travail est considéré comme accident du travail, peut être combattu par la preuve d'une origine ou d'une cause totalement étrangère au travail».

Cette preuve ne peut résulter d'une faute du salarié constituée par l'importance de son taux d'alcoolémie, en raison de l'obligation de contrôle et de surveillance incombant à l'employeur.

La thèse du suicide qui permet d'affirmer que le travail n'a joué aucun rôle dans la réalisation de l'acte ne peut être retenue car aucun élément de la cause ne permet d'affirmer que la victime se soit volontairement don- 
née la mort.

On voit qu'il y a une volonté de faire bénéficier les victimes des avantages attachés à l'indemnisation au titre des accidents de travail.

Quelques définitions sont essentielles à la bonne compréhension de la démonstration :

- la faute inexcusable (faute volontaire d'une exceptionnelle gravité) :

- de l'employeur : permet une indemnisation complémentaire de la victime et des ayants-droits

- de la victime : entraîne une réduction de son indemnisation (voir une suppression en cas d'accident de la circulation)

- la faute intentionnelle (faute volontaire avec intention délibérée de causer un dommage à la différence de la faute inexcusable) :

- de l'employeur : entraîne l'indemnisation de la victime et ayants-droits au titre des accidents du travail et un recours de la Sécurité Sociale au remboursement contre l'employeur.

- de l'employé : supprime toute indemnisation de son auteur - victime et ayants-droits - au titre des accidents du travail, et permets à la victime - non auteur - et ses ayants-droits un recours contre l'employeur, responsable des fautes de ses employés et permets le recours de la Sécurité Sociale à l'encontre exclusivement de l'auteur de l'accident - employé.

- la faute exclusive de l'employé exonère l'employeur de sa responsabilité pénale et si seulement si, elle a été la cause unique et exclusive de l'accident.

On examinera trois hypothèses au regard des responsabilités et de l'indemnisation des victimes et ayantsdroits :

1/ L'accident mortel de la circulation (sur la voie publique) ayant pour origine un véhicule conduit par un employé dans un état alcoolique ou sous l'emprise de cannabis.

2/ L'accident mortel ayant pour origine la conduite d'un engin de chantier par un employé ivre ou drogué.

3/ La chute mortelle d'un employé sous alcool ou drogue au regard de l'indemnisation de sa conjointe.

\section{Application des cas précé- demment exposés}

1 - Un employé conduit un véhicule de l'employeur sur une voie ouverte à la circulation publique (qui n'est pas un engin de chantier) en état d'ivresse ou sous l'emprise de cannabis et provoque un accident mortel :

Deux questions se posent : (a) les ayants-droits du conducteur seront-ils indemnisés par l'employeur ? (b) Les ayants-droits du passager seront-ils indemnisés par l'employeur ?

Concernant l'indemnisation des ayants-droits du conducteur : le fait de conduire un véhicule après une consommation excessive d'alcool - et de la même façon après consommation de cannabis - est considéré comme une faute inexcusable. En l'espèce, la loi du $5 / 7 / 85$ dite loi Badinter entre en application en concours avec la législation professionnelle des accidents du travail.

Les conséquences sont les suivantes : s'agissant d'un accident de la circulation routière (sur la voie publique), la faute inexcusable du conducteur (victime) et qui est la cause exclusive et unique de l'accident supprime tout droit à indemnisation. De plus, cette faute exclusive supprime la responsabilité de l'employeur. En outre, les victimes «par ricochet» subissent les conséquences de la faute commise par le conducteur, en application de l'article 6 loi Badinter (5/7/85) qui leur est opposable. Aussi, les ayants-droits sont dépourvus de recours contre l'employeur. Toutefois, une indemnisation réduite pourrait être obtenue par les ayants-droits en démontrant qu'une autre chose (autre véhicule ou faute de l'employeur) a participé à la réalisation de l'accident.

L'indemnisation des ayants-droits du passager peut s'envisager de la façon suivante : si l'on considère que le passager a lui-même commis une faute inexcusable par le fait d'avoir accepté de monter dans le véhicule et de s'exposer à un danger dont il aurait dû avoir conscience, l'indemnisation des ayants-droits du passager sera réduite, eu égard au partage des responsabilités. L'employeur ne pourra être recherché ni civilement ni pénalement sauf à démontrer qu'il a concouru à la réalisation de l'accident du fait de l'état du véhicule ou pour ne pas avoir pris les mesures nécessaires à l'égard de l'état de l'employé conducteur.

2 - Un employé conduisant un engin de chantier dans un état alcoolique ou sous emprise de cannabis écrase un autre employé. Qui est responsable ? Considérant qu'il s'agit d'un engin utilisé sur le chantier, c'est la législation relative aux accidents du travail qui s'applique, à l'exclusion de la loi Badinter. Il sera reproché à l'employeur de ne pas avoir pris toutes les mesures, au titre de son obligation de surveillance et de son pouvoir disciplinaire, pour s'opposer à cet état de fait. Pour s'exonérer, l'employeur ne peut se limiter à démontrer que son employé était ivre ou drogué. S'il démontre que :

- la faute inexcusable de son employé est seule à l'origine exclusive de l'accident : cela l'exonèrera pénalement 
- en outre, l'employé est soustrait à son autorité : cela lui permettra d'éviter tout recours et supprimera toute indemnisation des ayants-droits au titre des accidents du travail.

Mais cette preuve est difficile à établir car les tribunaux ne l'admettent que très rarement.

En conséquence, à défaut de cette difficile démonstration, l'employeur, ou son délégataire, sera pénalement sanctionné ainsi que l'employé lui-même se trouvant à l'origine de l'accident. Dans cette hypothèse, l'indemnisation de l'employé blessé ou des ayants-droits de la victime sera prise en charge au titre des accidents du travail.
3 - La conjointe d'un ouvrier faisant une chute mortel-

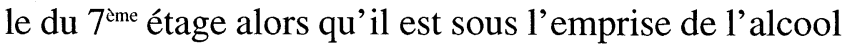
ou du cannabis recevra-t-elle une indemnité de l'employeur ? L'employé a commis une faute qualifiée d'inexcusable mais non intentionnelle (puisqu'il n'a pas la volonté du dommage ou alors c'est un suicide). Dès lors, son épouse (et ses ayants-droits) ne bénéficieront que d'indemnités réduites par la Sécurité Sociale. Mais s'ils parviennent à démontrer que cette faute n'est pas seule et exclusivement à l'origine du dommage, l'employeur pourra dès lors être recherché tant pénalement que pour l'obtention d'une indemnité complémentaire - par exemple si la chute n'a pu être évitée du fait de l'absence des mesures de sécurité (absence de harnais ou de dispositif de sécurité de chantier). 